\title{
Spin pumping and inverse spin Hall effect in Platinum and other $5 d$ metals: The essential role of spin-memory loss and spin-current discontinuities at interfaces
}

\author{
J.-C. Rojas-Sánchez ${ }^{\mathrm{a}}$, N. Reyren ${ }^{* a}$, P. Laczkowski ${ }^{\mathrm{a}}$, W. Savero ${ }^{\mathrm{b}}$, J.-P. Attanéb ${ }^{\text {, C. Deranlot }}{ }^{\mathrm{a}}$, \\ S. Gambarellib ${ }^{\mathrm{b}}$, M. Jamet ${ }^{\mathrm{b}}$, J.-M. George ${ }^{\mathrm{a}}$, L. Vila ${ }^{\mathrm{b}}$, and H. Jaffrès ${ }^{\mathrm{a}}$ \\ ${ }^{a}$ Unité Mixte de Physique CNRS/Thales and Université Paris-Sud 11, 91767 Palaiseau, France \\ ${ }^{\mathrm{b}}$ INAC/SP2M, CEA-Université Joseph Fourier, 38054 Grenoble, France
}

\begin{abstract}
It was recently realized that the spin Hall effect (SHE) can be very useful in the area of spintronics, due to its ability to generate pure spin current from charge current, without the use of any magnetic materials or magnetic field. The maximum conversion factor is given by the spin Hall angle $\theta_{\mathrm{SH}}$, which can take rather important values (above $10 \%$ in absolute value was reported for $\beta-\mathrm{Ta}$ and $\beta-\mathrm{W}$ ). This phenomenon is usually observed in materials with large spin-orbit coupling, either intrinsic $(\mathrm{Pt}, \mathrm{Ta}, \mathrm{W}$, etc.) or induced by heavy impurities $(\mathrm{Cu}$ doped with $\mathrm{Bi}$ or $\mathrm{Ir})$. To investigate this property, several groups studied the reciprocal effect, the so-called inverse spin Hall effect (ISHE), converting a pure "pumped" spin current into a charge current (measured by voltage detection in an "open circuit"). We focus here on the $5 d \mathrm{Pt}$ material. Values published nowadays for $\theta_{\mathrm{SH}}$ in Pt are scattered over one order of magnitude, with a clear correlation between the spin diffusion length $\ell_{\mathrm{sf}}$ and the $\theta_{\mathrm{SH}}$, both quantities being related to the spin-orbit strength or its inverse. We performed measurements of spin pumping in a cavity and measured the resulting ISHE voltage. We propose a model including spincurrent discontinuity or spin memory loss at the interfaces that may reconcile all the different observations. In particular, we demonstrate consistent values of spin diffusion length $\left(\ell_{\mathrm{sf}}=3.4 \pm 0.4 \mathrm{~nm}\right)$ and spin Hall angle $\left(\theta_{\mathrm{SH}}=0.056 \pm 0.010\right)$ for Pt in different metallic multilayer systems.
\end{abstract}

Keywords: spin Hall effect (SHE), spin pumping, spin memory loss, spin diffusion, metallic interface, ferromagnetism, spin-orbit, $5 d$ metals, ferromagnetic resonance.

\section{STATE OF THE ART: ONE ORDER OF MAGNITUDE DISPERSION IN PLATINUM SPIN DIFFUSION LENGTH AND SPIN HALL ANGLE}

The spin Hall effect is mostly studied in heavy metals which have an incomplete $4 d$ or $5 d$ orbital occupancy, such as $\mathrm{Ta}^{1,2}$, $\mathrm{W}^{3}$ or $\mathrm{Pt}^{4-13,14,2,15-18}$. The latter one has been extensively studied because of its chemical stability and probably also because it generates a perpendicular anisotropy on some $3 d$ metals such as Co. We will focus this article on the case of Pt, but the conclusion may be applied to any spintronics interface.

Once the data gathered from the literature ${ }^{4-13,14,2,15-18}$ for the spin Hall angle $\theta_{\mathrm{SH}}$ and spin diffusion length $\ell_{\mathrm{sf}}$ in $\mathrm{Pt}$ is graphically plotted, a general trend in the scattered data can be observed: In Fig.1a, a line reveal a constant $\theta_{\mathrm{SH}} \cdot \ell_{\mathrm{Sf}}$ product. This is explained by the fact that in combined spin pumping - ISHE experimental techniques, the expression allowing $\theta_{\mathrm{SH}}$ to be extracted involves both the physical parameters $\theta_{\mathrm{SH}}$ and $\ell_{\mathrm{sf}}$ only by their common product, meaning that both quantities are fully entangled and that one of them (usually $\ell_{\mathrm{sf}}$ ) has to be determined independently (see for instance Refs. 8 or 19). In section 5, we show that the spin diffusion length can be deduced from thickness-dependent measurements, but non-local spin valves and other techniques are also used. ${ }^{20}$ For many of the reported values, authors did not measure independently $\ell_{\text {sf }}$, or neglected some spin-flip at interfaces, leading to unreliable estimation of $\theta_{S H}$. The spin diffusion length as a function of the resistivity is shown in Fig.1b for the same group of article than Fig.1a. Only very weak correlation is observed. Nguyen $e t a l,{ }^{21}$ evidenced, after selection and correction of the residual resistivity of impurities, a much clearer trend between the conductivity and the spin diffusion length: The product ${ }^{21} \rho \cdot \ell_{\mathrm{sf}} \cong 0.9 \cdot 10^{-15} \Omega \mathrm{m}^{2}$ is about constant. That is also confirmed with ab-initio calculations ${ }^{22}$, showing $\rho \cdot \ell_{\mathrm{sf}}=0.61 \pm 0.02 \cdot 10^{-15} \Omega \mathrm{m}^{2}$. We call this quantity the spin resistance $r_{s}$, and we found in our case ${ }^{14}$ a value of $r_{s}=\rho \cdot \ell_{\mathrm{sf}}=0.59 \pm 0.07 \cdot 10^{-15} \Omega \mathrm{m}^{2}$. Note

*nicolas.reyren@thalesgroup.com 
that such a linear relationship is indeed expected in a Drude model of electronic conduction with the extra ingredient of a spin flip that occurs at each elastic collision with a given probability: As the conductivity is proportional to the mean free path, it will scale linearly with $\ell_{\text {sf. }}$ It is also important to note that interface scattering ${ }^{23,24}$ may play a preeminent role in the value of the measured resistance when the mean free path compares to the thickness of the films, which is the case in most of the present studies in Fig.1. Structural characterization would hence be useful in order to understand the source of the observed differences.

The scattering of the published data shows how important and not trivial is the estimation of proper material properties that are necessary to compare and evaluate the spin Hall effect of different experiments. Note that the dispersion may also result from a "real" dispersion of Pt properties related to the quality of the thin films and their interfaces. Resistivity probably gives a hint about sample quality ${ }^{21}$.
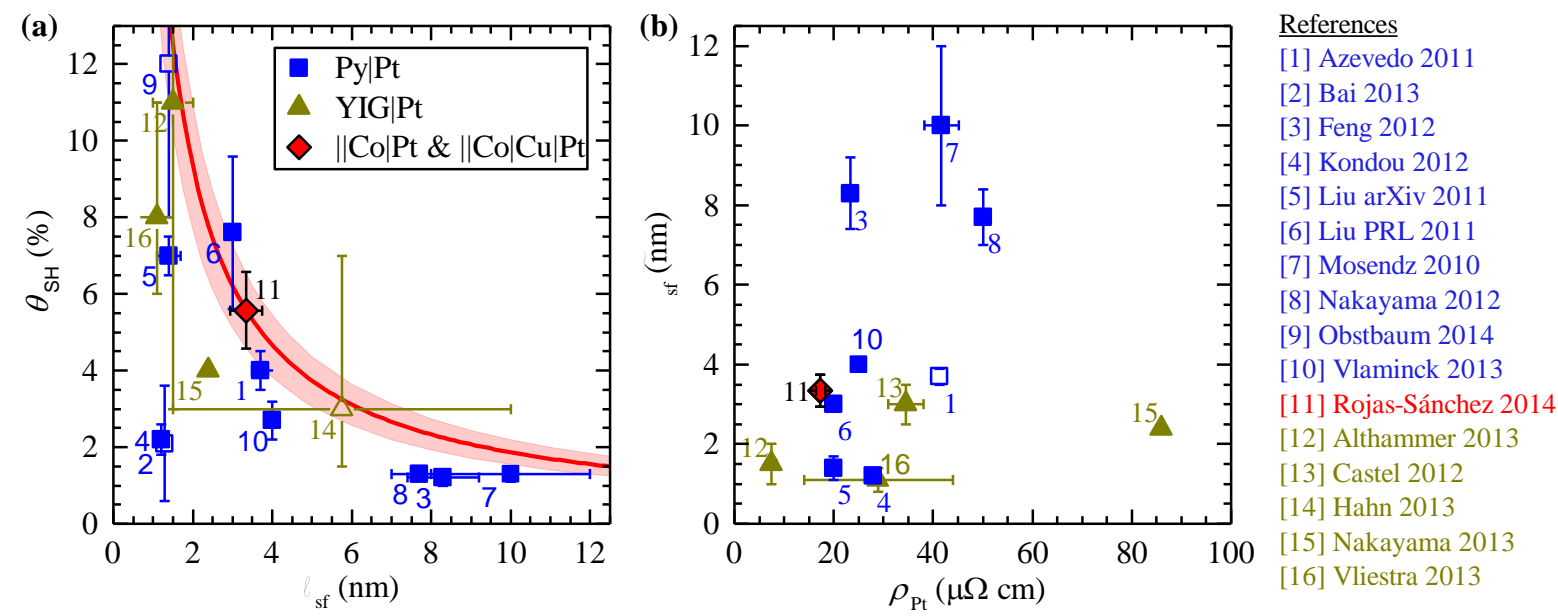

Figure 1: Literature reported values for Pt properties: (a) spin Hall angle as a function of the spin diffusion length, and (b) the spin diffusion length as a function of the resistivity. Hollow symbols in panel a (panel b) indicate that the spin diffusion length (the resistivity) was not directly measured by the authors. The red line in panel (a) corresponds to a constant product $\theta_{\mathrm{SH}}$. $\ell_{\text {sf }}=0.188 \mathrm{~nm}$.

\section{MATERIAL PROPERTIES: GROWTH, STRUCTURE AND RESISTIVITY}

For the series of experiments presented hereafter, we grew thin metallic films by dc magnetron sputtering in a single deposition chamber on thermally oxidized $\mathrm{Si}$ wafers $(400 \mathrm{~nm} \mathrm{SiO} 2)$, using high purity (4N) metals and Ar gas. Two series of samples were deposited: $\| \operatorname{Co}(15) \mid \operatorname{Pt}(t)$ and $\| \operatorname{Co}(15)|\operatorname{Cu}(5)| \operatorname{Pt}(t)$, where the number in parenthesis are thicknesses in nanometers and "|l" denotes the position of the substrate. We varied the thickness $t$ of the Pt layer from $2 \mathrm{~nm}$ to $50 \mathrm{~nm}$.

The samples are checked by low-angle X-ray reflectivity (XRR) using standard laboratory X-ray diffractometer (Bruker D8). Fitting ${ }^{\dagger}$ of the XRR spectrum allows the thickness and the roughness of the interfaces to be determined with a high precision. Typical XRR spectra with their fit are displayed in Fig.2a. The fitting process was done fixing the density of the materials, and letting free the thickness and the roughness among other parameters. It clearly shows that the roughness is about constant in the different types of films, and in particular is independent of the thickness of $\mathrm{Pt}$, as well as of the insertion of the $\mathrm{Cu}$ layer.

Resistance was measured on each sample either by van der Pauw method ${ }^{25}$ with contacts in the corners of rectangular pieces of samples (typically a few mm wide) or in patterned Hall bar structures of different sizes $\left(600 \times 40 \mu \mathrm{m}^{2} \mathrm{or}\right.$ $1 \mathrm{x} 0.1 \mathrm{~mm}^{2}$ ). Both techniques give the same results. Supposing a constant conductivity for $\mathrm{Pt}$, and assuming a simple parallel resistors model ${ }^{11}$ (see also Fig.5c), the measured total sheet conductance $G_{S}$ of the samples is simply given by $G_{S}=G_{S}^{0}+\sigma(\mathrm{Pt}) \cdot t$, where $\sigma(\mathrm{Pt})$ is the conductivity of $\mathrm{Pt}$ and $G_{S}^{0}$ is the sheet resistance of the $15 \mathrm{~nm}$ thick Co layer (plus the $5 \mathrm{~nm}$ thick $\mathrm{Cu}$ layer) that is, in principle, constant for all the $\| \operatorname{Co}(15) \mid \operatorname{Pt}(t)$ series (respectively the $\| \operatorname{Co}(15)|\operatorname{Cu}(5)| \operatorname{Pt}(t)$ series). In the simple framework of this model, the total sheet conductance should grow linearly with the thickness with a slope corresponding to the conductivity $\sigma(\mathrm{Pt})=1 / \rho(\mathrm{Pt})$. One can see in Fig.2b that Pt keeps the same conductivity in

\footnotetext{
$\dagger$ We used the commercial X'Pert Reflectivity software, version 1.3 by PANalytical B.V., Almelo, The Netherlands.
} 
both systems, and with thicknesses between two and fifty nanometers. This last result is even surprising, because interfaces should scatter the carriers as it was observed in other studies ${ }^{23,24}$. One can imagine that if the $\mathrm{Co} \mid \mathrm{Pt}$ or the $\mathrm{Cu} \mid \mathrm{Pt}$ interfaces are relatively transparent (for charges), then the scattering interface is found much lower at the $\mathrm{SiO}_{2} \mid \mathrm{Co}$ interface. As displayed in Fig.2b, the measured resistivity found by fitting both data series with the same conductivity (but different $G_{S}^{0}$ ) is $\rho(\mathrm{Pt})=17.3 \pm 0.6 \mu \Omega \mathrm{cm}$. This value is on the lowest limit of the reported room temperature values, hopefully indicating a good Pt quality.

The constancy of the Pt properties with its thickness is absolutely crucial in order to perform the analysis in the next sections. This analysis is indeed only valid if Pt keeps the same physical properties!
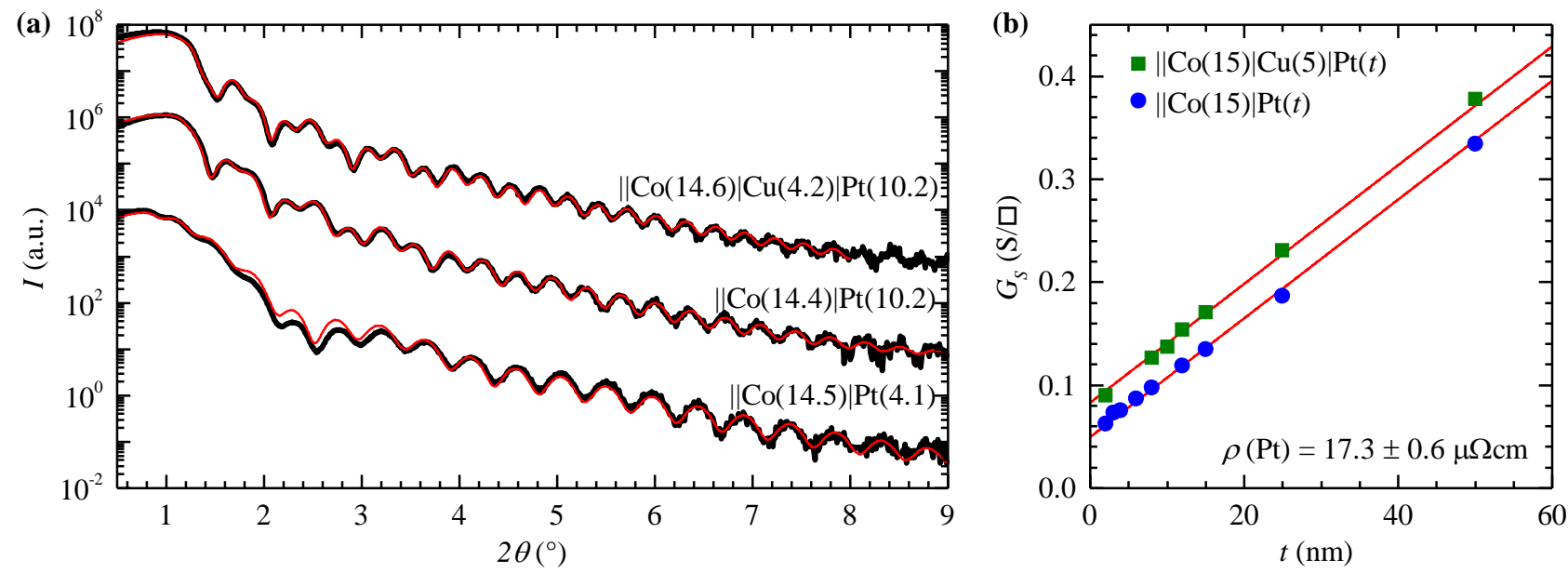

Figure 2: Characterization of the multilayers. (a) X-ray reflectivity (thick black curves) measured on three representative samples. The data is fitted (red thin curve) and parameters such as roughness are extracted and thicknesses are estimated. In the illustrated case, roughness has the following values: $0.64,0.74$ and $0.68 \mathrm{~nm}$ for $\mathrm{Cu}(5)|\operatorname{Pt}(10), \operatorname{Co}(15)| \operatorname{Pt}(10)$ and $\mathrm{Co}(15) \mid \mathrm{Pt}(4)$ interfaces, respectively; and at the interface with air is respectively $0.27,0.21$ and $0.24 \mathrm{~nm}$. The measured thicknesses of each layer appear in the figure (in $\mathrm{nm}$ ). (b) Total sheet conductance as a function of the Pt thickness for both series of sample, with and without $\mathrm{Cu}$ insertion. The data are fitted with a linear function, constraining the same slope, which is directly the conductivity.

\section{FERROMAGNETIC RESONANCE - CHARACTERIZATION OF THE COBALT LAYER}

In the present study, we use the ferromagnetic resonance (FMR) as a source of spin accumulation in the ferromagnettic layer, which will generate, in turn, a spin current towards the so-called "spin sink" by contact (spin diffusion). One must take into account, hereafter, the backflow mechanism. Studying the FMR allows us to access several material properties of the ferromagnetic layer (here $\mathrm{Co}$ ), that are necessary in order to properly estimate the spin current.

First, the measurement of the linewidth of the FMR curve as a function of the frequency $f$ of the radio-frequency (rf) excitation allows the magnetic damping $\alpha$ to be determined, as well as the frequency independent inhomogeneous contributions to the linewidth $\Delta H_{0}$ :

$$
\Delta H_{p p}=\Delta H_{0}+\frac{2}{\sqrt{3}}\left(\frac{\omega}{\gamma}\right) \alpha,
$$

where $\Delta H_{p p}$ is the peak-to-peak linewidth of the FMR absorption as a function of the magnetic field, taken as the magnetic field difference between the two extrema of the derivative of the absorption (and hence the numerical factor $2 / \sqrt{3}$ that relates to the full width at half maximum of an ideal Lorentzian curve, see Fig.3a); $\omega=2 \pi f$ is the pulsation of $h_{r f}$ and $\gamma=g \mu_{B} / \hbar$ is the gyromagnetic ratio, $g, \mu_{B}$, and $\hbar$ being the Landé factor, the Bohr magneton and the reduced Planck constant respectively. The frequency dependence of the FMR linewidth is displayed in Fig.3b. The inhomogeneous contributions find their origin in impurities, structural defects, oxidation or inhomogeneous thickness of the ferromagnetic film and increase the value not only of $\Delta H_{0}$ but also of the magnetic damping $\alpha$. The low values of $\Delta H_{0}$ that we find is an indication of the relatively ${ }^{26-29}$ good quality of the ferromagnetic layer and allow us to consider the magnetic damping 
value free of inhomogeneities contributions. Thus, the damping itself probes the quality of the FM layer as well, but it also probes, in our case, the neighboring heavy metal layer that absorbs part of the generated spins, and hence increases the damping as we will discuss below.

Second, measuring the resonance frequency as a function of the external magnetic field allows the effective magnetization $M_{S}^{\text {eff }}$ to be estimated using the formula:

$$
\left(\frac{\omega}{\gamma}\right)^{2}=\left(B+\mu_{0} H_{K}\right)\left(B+\mu_{0} H_{K}+\mu_{0} M_{s}^{\text {eff }}\right)
$$

Note that this expression corresponds to the Kittel formula if the in-plane anisotropy $H_{K}$ is neglected or negligible as it is the case for the $15 \mathrm{~nm}$ Co layers in our samples (see Fig.3c). More details about the calculations and hypothesis can be found for example in Ref. 30.

With the above full characterization we can calculate the effective spin mixing conductance which reads ${ }^{4,31,32}$ :

$$
g_{\mathrm{eff}}^{\uparrow \downarrow}=\frac{4 \pi M_{\mathrm{eff}} t_{\mathrm{Co}}}{g \mu_{\mathrm{B}}}\left(\alpha_{\mathrm{Co}(\mid \mathrm{Cu}) \mid \mathrm{Pt}}-\alpha_{\mathrm{Co}}\right),
$$

where $\alpha_{\mathrm{Co}}$ stands for the magnetic damping intrinsic of Co layer, and $\alpha_{\mathrm{Co}(\mid \mathrm{Cu}) \mid \mathrm{Pt}}$ corresponds to the magnetic damping of $\| \mathrm{Co} \mid \mathrm{Pt}$ or $\| \mathrm{Co}|\mathrm{Cu}| \mathrm{Pt}$ system with the same thickness of $\mathrm{Co}, t_{\mathrm{Co}}$. The magnetic damping $\alpha_{\mathrm{Co}}$ was measured in a $\| \mathrm{Co}(15) \mid \mathrm{Al}(5)$ reference sample which is free of spin pumping effect ${ }^{14}$ (see section 5).

Taking in account the spin pumping and the ISHE, the spin current which results from FMR is estimated by ${ }^{4,10,33}$ :

$$
J_{S}^{\text {eff }}=\frac{2 e}{\hbar} \frac{g_{\mathrm{eff}}^{\uparrow \downarrow} \gamma^{2} \hbar h_{\mathrm{rf}}^{2}}{8 \pi \alpha^{2}} \frac{4 \pi M_{\mathrm{eff}} \gamma+\sqrt{\left(4 \pi M_{\mathrm{eff}} \gamma\right)^{2}+4 \omega^{2}}}{\left(4 \pi M_{\mathrm{eff}} \gamma\right)^{2}+4 \omega^{2}} .
$$
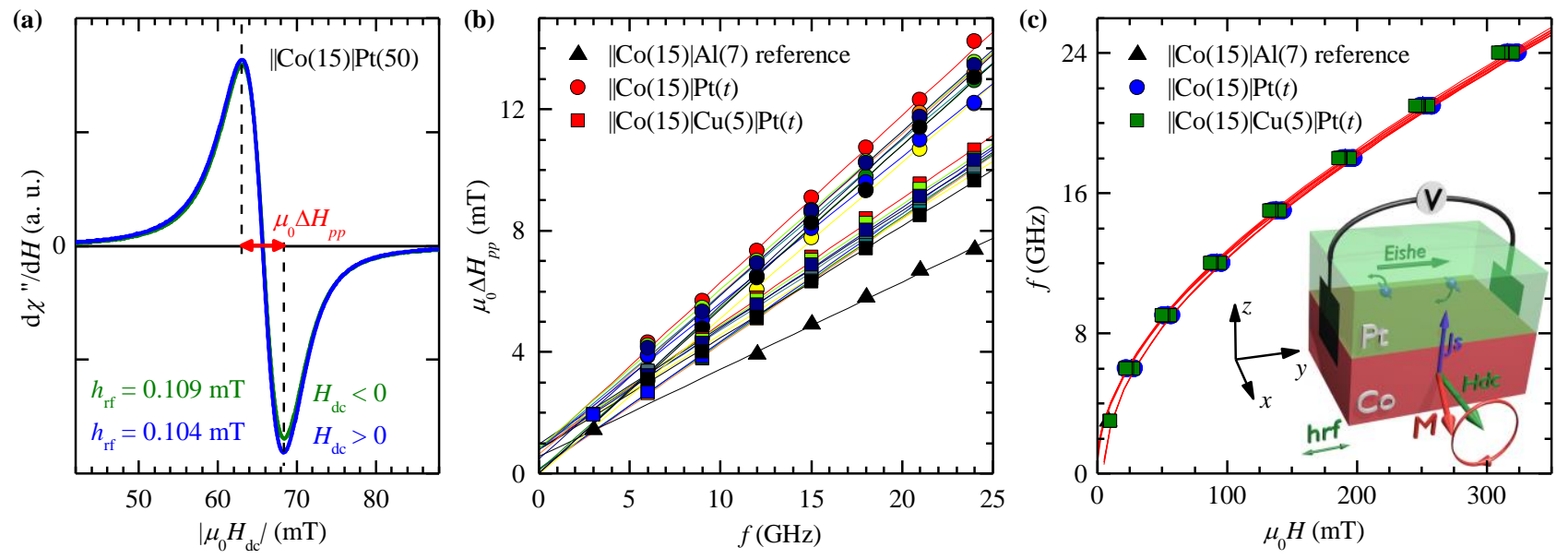

Figure 3: FMR measurements. (a) A typical ferromagnetic resonance spectrum showing the derivative of the absorption as a function of the applied magnetic field (positive in blue, negative in green, nearly undistinguishable). The peak-to-peak linewidth $\Delta H_{p p}$ is indicated. (b) Linear relationship (Eq.1) between the width of the FMR and the resonance frequency. Colors from red to dark blue indicate the thickness: There is no clear correlation with Pt thickness, except for $t=2 \mathrm{~nm}$ that has the largest $\Delta H_{0}$. (c) Illustration of the Kittel law relating the frequency to the magnetic field of the FMR (Eq.2). Inset: General configuration of the spin pumping from a ferromagnetic $(\mathrm{F})$ metal (here $\mathrm{Co}$ ) to a non-magnetic $(\mathrm{N})$ one (here $\mathrm{Pt}$ ).

\section{THEORITICAL MODEL OF THE INVERSE SPIN HALL EFFECT AND SPIN PUMPING INCLUDING THE "SPIN MEMORY LOSS"}

\subsection{Introducing the spin memory loss in the diffusion equations}

In this section, we follow the Valet-Fert diffusive model $^{34}$ which is well established for spin transport at metallic interface and magnetic multilayers. In such a model, for a steady-state one has:

$$
\nabla^{2} \mu_{s}=\frac{\mu_{s}}{\ell_{s f}^{2}},
$$

where $\mu_{s}=\mu_{\uparrow}-\mu_{\downarrow}$. The spin current which is carried by conduction electrons is then defined as

$$
j_{s}=-\frac{\hbar}{2 e} \frac{1}{e \rho} \nabla \mu_{s} \rightarrow \frac{e}{\hbar / 2} j_{s}=-\frac{1}{e \rho} \nabla \mu_{s}=-\frac{1}{e} \frac{\ell_{\mathrm{sf}}}{r_{s}} \nabla \mu_{s},
$$

where $j_{s}$ is expressed in $\mathrm{J} / \mathrm{m}^{2}$ and we used the definition of the spin resistance $r_{s}=\rho \cdot \ell_{\text {sf }}$ for the second equality which express the spin current in units of $\mathrm{A} / \mathrm{m}^{2}$. 
These equations can be used to model our ferromagnetic ('F')|non-magnetic ('N') interface. We consider now, based on former GMR studies ${ }^{35}$, that there exists spin memory loss (SML) at the $\mathrm{F} \mid \mathrm{N}$ interface, i.e. that there might be an interface region 'I', where spin relaxation occurs. The resulting physical system is modeled as sketched in Fig.4a. With the ansatz $\mu_{s}(z)=A_{k} \exp \left(\frac{z}{\ell_{\mathrm{sf}, k}}\right)+B_{k} \exp \left(-\frac{z}{\ell_{\mathrm{sf}, k}}\right)(k$ stands for I or $\mathrm{N}$ depending on the region), and using boundary conditions such that (1) the spin current out of $\mathrm{F}$ is $\frac{\partial \mu_{s}}{\partial z}(z=0)=-\frac{e j_{s 0}^{\text {eff }} r_{s I}}{\ell_{\mathrm{sf}, \mathrm{I}}}$, that (2) no current gets out at the non-magnetic boundary with the "air", and that both (3) the chemical potential and (4) its spatial derivative (the spin current) must be continuous at the $\mathrm{I} \mid \mathrm{N}$ interface. The solution is ${ }^{14}$ :

$$
\left\{\begin{array}{ll}
\mu_{s}(z)=C \cdot r_{s \mathrm{~N}}^{\infty} \cosh \left(\frac{t_{\mathrm{I}}-z}{\ell_{\mathrm{sf}, \mathrm{I}}}\right) \cosh \left(\frac{t_{\mathrm{N}}}{\ell_{\mathrm{sf}, \mathrm{N}}}\right)+r_{s \mathrm{I}} \sinh \left(\frac{t_{\mathrm{I}}-z}{\ell_{\mathrm{sf}, \mathrm{I}}}\right) \sinh \left(\frac{t_{N}}{\ell_{\mathrm{sf}, N}}\right) & \mid 0 \leq z \leq t_{\mathrm{I}} \\
\mu_{s}(z)=C \cdot r_{s \mathrm{~N}}^{\infty} \cosh \left(\frac{t_{\mathrm{I}}+t_{\mathrm{N}}-z}{\ell_{\mathrm{sf}, \mathrm{N}}}\right) & \mid t_{\mathrm{I}} \leq z \leq t_{\mathrm{N}}
\end{array},\right.
$$

where $C=e j_{s 0}^{\text {eff }} r_{s I}\left(r_{s \mathrm{~N}}^{\infty} \cosh \left(t_{\mathrm{N}} / \ell_{\mathrm{sf}, \mathrm{N}}\right) \sinh (\delta)+r_{s \mathrm{I}} \sinh \left(t_{\mathrm{N}} / \ell_{\mathrm{sf}, \mathrm{N}}\right) \cosh (\delta)\right)^{-1}$. A solution is illustrated in Fig.4a, next to the schematic structure. The spin current is then obtained taken the spatial derivative as shown in Eq.6. By substituting $t_{\mathrm{I}} / \ell_{\mathrm{sf}, \mathrm{I}}$ by $\delta$ and taking the ratio of the spin current reaching the $\mathrm{N}$ layer by the spin current effectively emitted by $\mathrm{F}$ (considering the back-flow), one gets ${ }^{14}$ :

$$
R_{\mathrm{SML}}=\frac{j_{S}\left(z=t_{\mathrm{I}}\right)}{j_{S}(z=0)}=\frac{r_{S I}}{r_{S \mathrm{~N}}^{\infty} \operatorname{coth}\left(\frac{t_{\mathrm{N}}}{\ell_{\mathrm{Sf}, \mathrm{N}}}\right) \sinh (\delta)+r_{S I} \cosh (\delta)} .
$$

This ratio indicates what fraction of the spin current pumped out of $\mathrm{F}$ effectively reaches $\mathrm{N}$ where it can be converted in a charge current by the ISHE. The value of this ratio for different parameters is graphically shown in Fig.4.The effective reduction of spin current is larger for thinner films, due to the flattening of $\mu_{s}$. In the case of the Pt|Co interface, RSML is essentially constant above 2-3 nm, explaining why the observed damping Pt thickness variation is so weak, as we will see later.
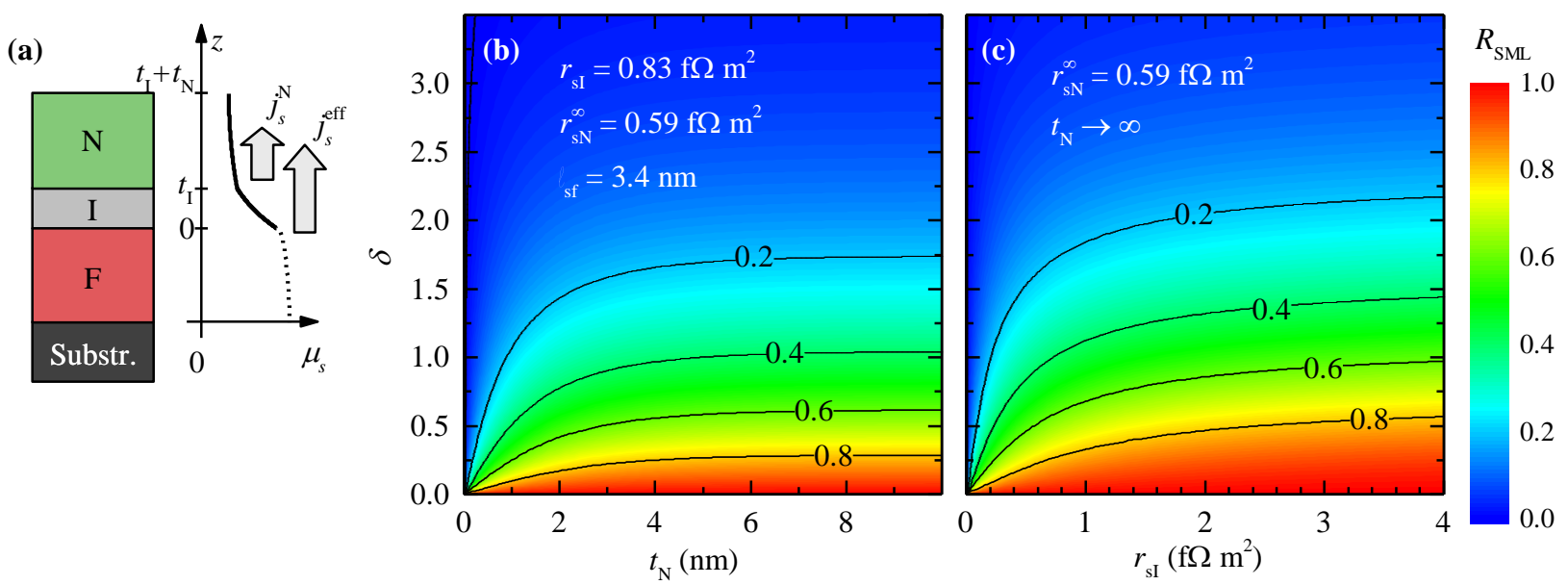

Figure 4: Illustration of our model of a ferromagnetic ' $\mathrm{F}$ '|non-magnetic ' $\mathrm{N}$ ' bilayer including the spin memory loss at their interface 'I'. (a) If 'I' is considered, a new term must be considered, the spin memory loss. The expected profile of spin accumulation is schematized on the side of the sample structure. The drop of $\boldsymbol{\mu}_{\boldsymbol{s}}$ in the 'I' region corresponds to spin memory loss. The spin current (derivative of $\mu_{s}$ ) inside $\mathrm{N}$ is much reduced. The currents corresponding to the chemical potential are indicated. The interface F|substrate is ignored in our model. (b) $R_{\mathrm{SML}}=J_{S}^{N} / J_{S}^{\text {eff }}$ (color scale) as a function of the non-magnetic metal thickness $t_{\mathrm{N}}$ and the spin-flip parameter $\delta, R_{\mathrm{SML}}=1$ indicating a completely transparent interface, and $R_{\mathrm{SML}}=0$ an interface which absorbs all the spin current. The other parameters indicated in the figure are the one corresponding to the Pt|Co system. (c) In the limit of very large $\mathrm{N}$ films $\left(t_{\mathrm{N}} \gg \ell_{\mathrm{Sf}}\right), R_{\mathrm{SML}}$ as a function of the interface spin resistance $r_{\mathrm{sI}}$ and $\delta$, fixing the $\mathrm{N}$ with the Pt parameters as indicated in the figure.

In the case of a trilayer $\mathrm{F}|\mathrm{NM} 1| \mathrm{NM} 2$ such as our $\mathrm{Co}|\mathrm{Cu}| \mathrm{Pt}$, two interfaces must be considered, resulting in a model system F|I1|NM1|I2|NM2. The effective interface spin memory loss is not the simple sum of the SML in each interface, but rather ${ }^{14}: \cosh \left(\delta^{\text {eff }}\right)=\cosh \left(\delta_{2}\right)\left(\cosh \left(\delta_{1}\right)+r_{s I 1} / r_{s I 2} \sinh \left(\delta_{1}\right)\right)$, and for the effective spin resistance: $\left(r_{s I}^{\text {eff }}\right)^{-1}=$ $r_{s I 2} \sinh \left(\delta_{2}\right) / \sinh \left(\delta^{\mathrm{eff}}\right)\left(\cosh \left(\delta_{1}\right)+r_{s \mathrm{I} 2} / r_{s 11} \sinh \left(\delta_{1}\right)\right)$. 


\subsection{Consequences of the SML for the damping}

What are the consequences of the SML for the theoretical expression of the damping? Considering the spin pumping theory in the limit $\omega \ll 1 / \tau_{\mathrm{sfN}, \mathrm{N}}$, and neglecting the imaginary part of the spin mixing conductance that we can take generally small between metals ${ }^{36}$, the effective spin mixing conductance is ${ }^{4,11,31,32}$ :

$$
g^{\uparrow \downarrow, \text { eff }}=\frac{g^{\uparrow \downarrow}}{1+\tilde{g}^{\uparrow \downarrow} \beta} \quad,
$$

where $\tilde{g}^{\uparrow \downarrow}$ is such that $\frac{2 e^{2}}{\hbar} \tilde{g}^{\uparrow \downarrow} r_{s N}^{\infty}=\frac{1}{2} \sqrt{3 / \epsilon}, \epsilon$ is the ratio of the elastic to the spin-flip times, taking a value of 0.1 for Pt, and $\beta$ is the back flow factor. Taking in account the profile of the chemical potential with the SML (Eq.7), one can express $\beta$ as a function of the material parameters (including the SML). Finally one can find the following expression for the increase of the damping that takes in account the back-flow and the SML ${ }^{14}$ :

$$
\Delta \alpha=\frac{g \mu_{B}}{4 \pi M_{\mathrm{eff}} t_{\mathrm{F}}} g^{\uparrow \downarrow, \text { eff }}=\frac{g \mu_{B}}{4 \pi M_{\mathrm{eff}} t_{\mathrm{F}}} g^{\uparrow \downarrow} \frac{r_{\mathrm{SI}} \cosh (\delta)+r_{S \mathrm{~N}}^{\infty} \sinh (\delta) \operatorname{coth}\left(\frac{t_{\mathrm{N}}}{\ell_{\mathrm{sf}, \mathrm{N}}}\right)}{r_{S I}\left(1+\frac{1}{2} \sqrt{\frac{3}{\epsilon}} \operatorname{coth}\left(\frac{t_{\mathrm{N}}}{\ell_{\mathrm{Sf}, \mathrm{N}}}\right)\right) \cosh (\delta)+\left(r_{S \mathrm{~N}}^{\infty} \operatorname{coth}\left(\frac{t_{\mathrm{N}}}{\ell_{\mathrm{sf}, \mathrm{N}}}\right)+\frac{1}{2} \sqrt{\frac{3}{r_{S I}}} r_{S \mathrm{~N}}^{\infty}\right) \sinh (\delta)} .
$$

It appears clearly that the $\mathrm{N}$ thickness dependence of the damping variation is more complex than in the simple case of spintronics interfaces without SML or spin-current discontinuities where the scaling is $\tanh \left(t_{\mathrm{N}} / \ell_{\mathrm{sf,N}}\right)$. This means that it cannot be used as-it-is to determine the spin diffusion length of $\mathrm{N}$.

\subsection{Consequences of the SML for ISHE current}

Similarly, the usual expression for the ISHE charge current is modified:

$$
I_{C}=\theta_{\mathrm{SH}} \ell_{\mathrm{sf}} W J_{s} \tanh \left(\frac{t_{\mathrm{N}}}{2 \ell_{\mathrm{sf}}}\right) \rightarrow I_{C}=\theta_{\mathrm{SH}} \ell_{\mathrm{sf}} W J_{S}^{\text {eff }} \tanh \left(\frac{t_{\mathrm{N}}}{2 \ell_{\mathrm{sf}}}\right) \cdot R_{\mathrm{SML}} .
$$

One can readily see that the charge current will generally be under-estimated if SML is neglected $\left(0<R_{\mathrm{SML}}<1\right)$, the error being dependent on the details of the interface. That means that, ignoring the SML, different apparent spin Hall angle can be found for a single $\mathrm{N}$ metal when different type of interfaces are studied, either using a different ferromagnetic metal or adding an intermediate layer. In Fig.1a, as expected, all the publish measurements that are not considering the SML are below our $\theta_{\mathrm{SH}} \ell_{\mathrm{sf}}$ curve due to the rather low value of $R_{\mathrm{SML}}$.

\section{MEASUREMENTS AND RESULTS FOR PLATINUM}

\subsection{Damping measurements}

Damping measurements shown in Fig.5 were performed on the series of samples described in section 2, with the technique combining spin pumping and ISHE. For frequency-dependent measurements described in section 3, the sample in excited with a strip-line, but for spin-pumping-ISHE measurements, the sample was placed in a split-cylinder resonant cavity. As explain above, great care was used in order to center the sample inside the resonant cavity to avoid spurious effects. A straightforward analysis using the usual formula found without considering the spin memory loss would lead to a spin diffusion length shorter than $2 \mathrm{~nm}$. As it can be seen in the next sub-section, such value is incompatible with the thickness dependence of the ISHE current (or measured voltage). As we calculated, about $50 \%$ of the spins are lost at the interface which acts as a spin sink that increases the damping. This interface-related phenomenon does not depend on Pt thickness and hence cannot give clues on the value of the spin diffusion length in "bulk" Platinum. 

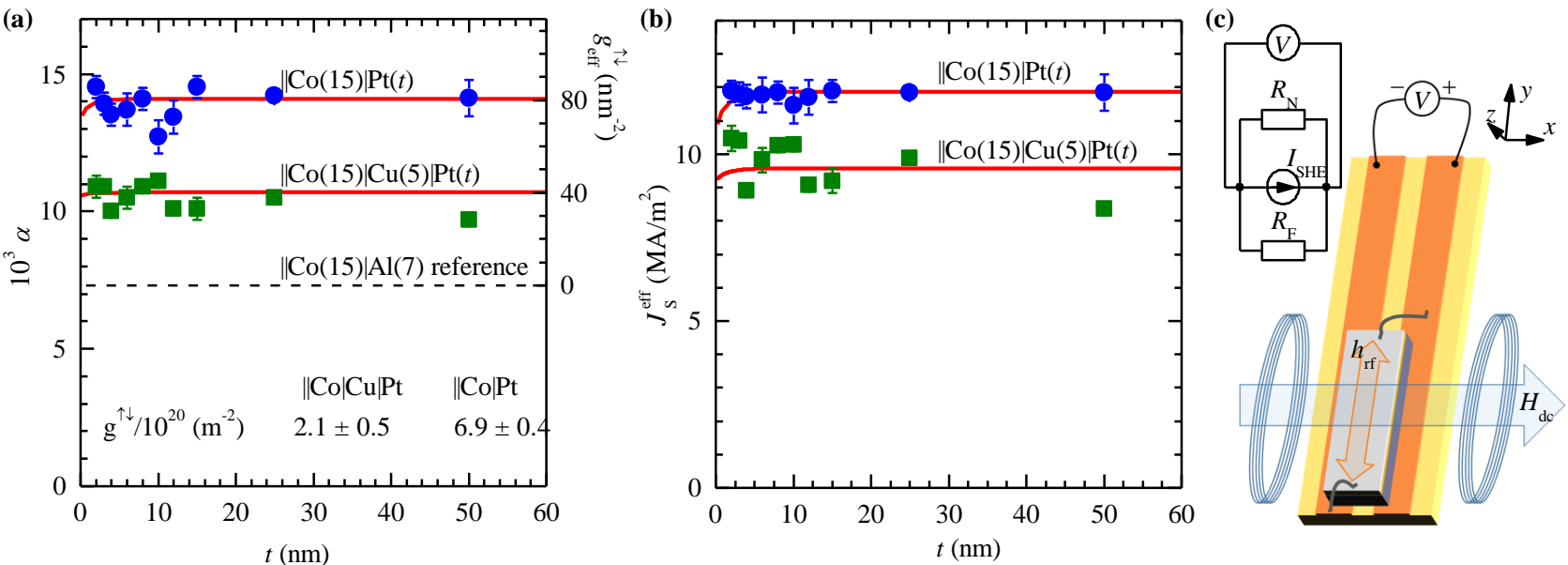

Figure 5: Results extracted from the FMR measurements on strip-line (for frequency dependence) and in cavity (for proper estimation of $h_{\mathrm{rf}}$ and large $Q$ ). (a) Platinum thickness dependence of the damping and the corresponding spin mixing conductance. The damping is essentially constant down to $2 \mathrm{~nm}$ of Pt. (b) Estimated effective spin current as calculated from Eq.4. The behavior of the effective spin current follows the one of the damping; no clear variation with thickness down to $2 \mathrm{~nm}$ is detectable. The fits (red lines) of both (a) and (b) are calculated using Eq. 10 which takes in account the spin memory loss. (c) Equivalent circuit used to analyze the data in the spin-pumping-ISHE configuration, and schematics of the used experimental setup. For practical reasons, the sample is glued on a PCB (yellow) with two Cu conducting lines (orange) that are connected by wire bonding (grey) at the edges of the sample. The sample and the PCB are inserted in a half-cylinder resonant cavity (not shown) that generates the rf magnetic field $h_{\mathrm{rf}}$. The whole system is placed inside large coils (blue) that generate the quasi-static magnetic field $H_{\mathrm{dc}} . H_{\mathrm{dc}}$ is swept at less than $500(\mathrm{~A} / \mathrm{m}) / \mathrm{s}$, and both the dc voltage $V$ and the absorption of the rf field are monitored simultaneously.

\subsection{ISHE voltage measurements and charge current production}

It is important to note that the amplitude of the rf magnetic field $h_{\mathrm{rf}}$ must be determined experimentally in order to be able to calculate properly the spin current density which allows to quantify the efficiency of spin current to charge current conversion due to ISHE in the present study. For such experiments we carried out a simultaneous measurement of FMR spectrum and dc transversal voltage (Fig.5c) in a cylindrical cavity resonance in the X-band (frequency $f$ around $9.7 \mathrm{GHz}$ ). We measure the quality factor $Q$ with the sample inside the cavity and then we calculate $h_{\mathrm{rf}}$ following the calibration conversion factor. For this experiment all the samples were cut in rectangular slabs of $W \times L=0.4 \times 2.4 \mathrm{~mm}^{2}$ then glued on a PCB holder with two $\mathrm{Cu}$ paths (Fig.5c). The samples edges along the $y$-axis are connected to the Cu paths by $\mathrm{Al}$ wirebonding. The rf field $h_{\mathrm{rf}}$ was about $80 \mathrm{~A} / \mathrm{m}( \pm 20 \%$ depending on the sample) for $200 \mathrm{~mW}$ of microwave power. Furthermore we performed the measurements in both cases: When $H_{\mathrm{dc}}$ is applied parallel to the film and when the sample is turned $180^{\circ}$, equivalent of reversing the sign of $H_{\mathrm{dc}}$ with respect to the first case. The value of $h_{\mathrm{rf}}$ marginally changes as the sample is rotated in the split-cylinder cavity, leading to slightly different quality factor due to the fact that it might be not rigorously centered. (Note that the sample must be rotated simply because the $H_{\mathrm{dc}}$ magnet source is unipolar.)

Thus we control the right placement of sample inside the cavity in order to reduce undesirable effects such as AHE, PHE or AMR, fitting the Lorentzian voltage at resonance with anti-symmetric and symmetric shapes. $V\left(H_{\mathrm{dc}}\right)$ curves for representative thicknesses are displayed in Fig.6a. Our best estimate of $V_{\text {ISHE }}$ is made by considering only the symmetric part, because it is believed that the asymmetric one is related to previously mention spurious effects, and the mean value (weighted by $h_{\mathrm{rf}}{ }^{2}$ ) is taken between positive and negative $H_{\mathrm{dc}}$. The resulting $V_{\mathrm{ISHE}}$ is then divided by the measured resistance (see equivalent circuit in Fig.5c) in order to calculate the ISHE charge current, $I_{\mathrm{c}}$, that is displayed in Fig.6b. The reduction of the system's resistance with Pt thickness explains that the measured voltages pass through a maximum for non-magnetic metal thickness of about twice the spin diffusion length, being minimal both for the zero thickness limit (no ISHE voltage) and the infinite one (zero resistance). 

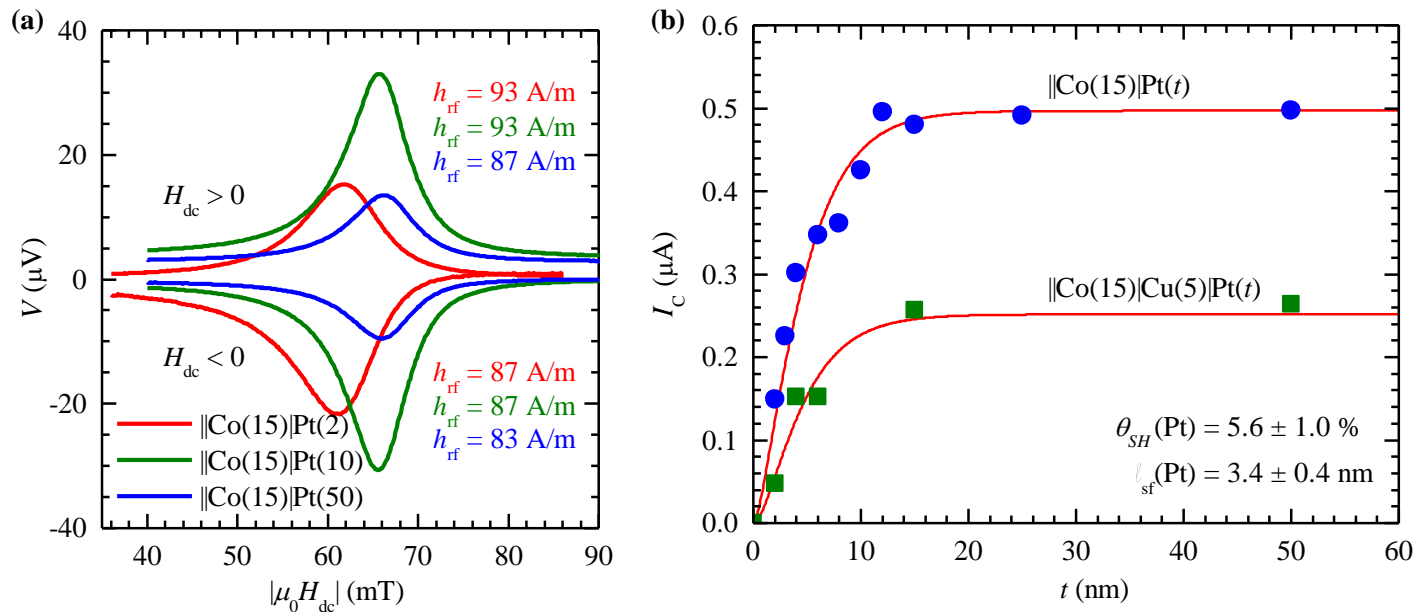

Figure 6: ISHE measurements. (a) ISHE voltage measured for different samples at positive and negative quasi-static magnetic field (signs according to setup as in Fig.5c). Note that the signal is mostly symmetric and is well reversed with the sign change of $H_{\text {dc. }}$ (b) Charge current as a function of the Pt thickness. Clear thickness dependence can be observed up to about $10 \mathrm{~nm}$. Concomitant fit of both data series give a spin Hall angle of 5.6 $\pm 1.0 \%$ and a spin diffusion length in Pt of $3.4 \pm 0.4 \mathrm{~nm}$.

In order to study the interface-related phenomena at play, for example to study a possible role of an induced magnetization of Pt that could modify the ISHE,,$^{37,38}$ or Rashba-related effects, we inserted a layer of $\mathrm{Cu}$ in-between $\mathrm{Pt}$ and $\mathrm{Co}$. This layer is about $5 \mathrm{~nm}$, which is much smaller than the spin diffusion length in $\mathrm{Cu}$, but large enough to prevent the induced magnetization of Pt by Co. This means that the spin current should be unaffected, and only the possible magnetization of Pt should be cancelled. As it can be seen in Fig.6b, this is not the case. We observe that (i) the thickness dependence seems to be the same (supporting a meaningful estimation of $\ell_{\mathrm{sf}}$ ), and (ii) the charge current changes by a factor of about two. If one tries to extract the spin Hall angle from these data with the standard formula, i.e. without the correction proposed in Eq. 8 and 11, one gets inconsistent values of $\theta_{\mathrm{SH}} \simeq 3.1 \%$ and $2.0 \%$ for the $\mathrm{Co} \mid \mathrm{Pt}$ or the $\mathrm{Co}|\mathrm{Cu}| \mathrm{Pt}$ systems respectively. Such change of a property, which should be related to the bulk Pt only, is unexpected.

The simplest explanation may be that interfaces may present spin-current discontinuities and spin memory loss. ${ }^{14,35,40}$ In particular, a larger spin memory loss or spin-current discontinuity, as invoked in recent investigations ${ }^{22}$, will reduce the efficiency of spin-injection in Pt. This would lead to a smaller charge current through ISHE. Using spin memory loss parameters reported in the literature ${ }^{14,21,40-42}$ and calculated (see table 1), as well as the magnetic properties of Co measured with the damping, one can consistently fit both data set, with and without $\mathrm{Cu}$ insertion using an unique spin Hall angle for Platinum: $\theta_{\mathrm{SH}}(\mathrm{Pt})=0.056 \pm 0.010$.

Table 1. Table of the spin memory loss parameters used for the analysis.

\begin{tabular}{|c|c|c|c|c|}
\hline & $\mathrm{Co} \mid \mathrm{Cu}^{40}$ & $\mathrm{Cu} \mid \mathrm{Pt}^{41}$ & $\mathrm{Co} \mid \mathrm{Pt}^{21}$ & $\mathrm{Co}|\mathrm{Cu}| \mathrm{Pt}^{14}$ \\
\hline$\delta$ & 0.25 & 0.9 & 0.9 & 1.2 \\
\hline$r_{S \mathrm{I}}\left(\mathrm{f} \Omega \mathrm{m}^{2}\right)$ & 2 & 1.7 & 0.83 & 0.85 \\
\hline
\end{tabular}

\section{CONCLUSION AND PERSPECTIVES}

In conclusion, we showed that spin memory loss is a natural way to explain both qualitatively and quantitatively our results in different series of samples $\| \mathrm{Co} \mid \mathrm{Pt}$ and $\| \mathrm{Co}|\mathrm{Cu}| \mathrm{Pt}$. The spin memory loss and spin-current discontinuities have important consequences:

a) The intrinsic spin Hall angle of the non-magnetic material is underestimated if the spin memory loss is neglected. ${ }^{14,22}$

b) Using the non-magnetic thickness dependence of the damping to estimate the spin diffusion length leads to underestimation of this quantity. As a consequence, the spin Hall angle is over-estimated due to the entanglement of these parameters in the expression of the charge current. A trilayer treatment is generally required as exposed in this paper. ${ }^{14}$ 
These two points can largely explain the observed scattering of the reported values for Platinum, as displayed in Fig.1a. Indeed, studies underestimating the spin diffusion length will produce proportionally larger spin Hall angle: A measurement of current in a single sample only gives a product $\theta_{\mathrm{SH}} \cdot \ell_{\mathrm{sf}}$, in our case we have $\theta_{\mathrm{SH}} \cdot \ell_{\mathrm{Sf}}=0.188 \mathrm{~nm}$ (see Fig.1a). Interestingly, all the reported data points are below this line, due to the fact that spin memory loss was neglected up to now. We conclude from this observation that, although the Platinum intrinsic properties may vary from one study to the other, most of the data may be reconciled by both considering a better estimation of the spin diffusion length (not using the damping, but the charge current), and spin memory loss at the interfaces.

We show that interface effects play important roles. In the present case roughly half of the spin current is lost. Interface engineering may allow to better control spin memory loss and reduce it as much as possible, for example using metals with very transparent interface such as silver. These observations not only impact the fundamental studies, but also very practical devices, such as spin-transfer-torque MRAM and in general any system in which a spin current crosses an interface.

\section{ACKNOWLEDGMENTS}

We acknowledge U. Ebels, W. E. Bailey and G. Desfonds for technical support with the FMR measurements, J. Bass, A. Fert, and F. Freimuth for fruitful discussions. This work was partly supported by the French Agence Nationale de la Recherche (ANR) through Projects SPINHALL (2010-2013) and SOSPIN (2013-2016).

\section{REFERENCES}

[1] Liu, L., Pai, C.-F., Li, Y., Tseng, H. W., Ralph, D. C., Buhrman, R. A., "Spin-torque switching with the giant spin Hall effect of tantalum.," Science 336(6081), 555-558 (2012).

[2] Hahn, C., de Loubens, G., Klein, O., Viret, M., Naletov, V. V., Ben Youssef, J., "Comparative measurements of inverse spin Hall effects and magnetoresistance in YIG/Pt and YIG/Ta," Phys. Rev. B 87(17), 174417 (2013).

[3] Pai, C., Liu, L., Li, Y., Tseng, H. W., Ralph, D. C., Buhrman, R. A., "Spin transfer torque devices utilizing the giant spin Hall effect of tungsten," Appl. Phys. Lett. 101(12), 122404 (2012).

[4] Azevedo, A., Vilela-Leão, L. H., Rodríguez-Suárez, R. L., Lacerda Santos, A. F., Rezende, S. M., "Spin pumping and anisotropic magnetoresistance voltages in magnetic bilayers: Theory and experiment," Phys. Rev. B 83(14), 144402 (2011).

[5] Bai, L., Hyde, P., Gui, Y. S., Hu, C.-M., Vlaminck, V., Pearson, J. E., Bader, S. D., Hoffmann, A., "Universal Method for Separating Spin Pumping from Spin Rectification Voltage of Ferromagnetic Resonance," Phys. Rev. Lett. 111(21), 217602 (2013).

[6] Feng, Z., Hu, J., Sun, L., You, B., Wu, D., Du, J., Zhang, W., Hu, A., Yang, Y., et al., "Spin Hall angle quantification from spin pumping and microwave photoresistance," Phys. Rev. B 85(21), 214423 (2012).

[7] Kondou, K., Sukegawa, H., Mitani, S., Tsukagoshi, K., Kasai, S., "Evaluation of Spin Hall Angle and Spin Diffusion Length by Using Spin Current-Induced Ferromagnetic Resonance,” Appl. Phys. Express 5(7), 073002 (2012).

[8] Liu, L., Buhrman, R. A., Ralph, D. C., "Review and Analysis of Measurements of the Spin Hall Effect in Platinum," Mesoscale and Nanoscale Physics, arXiv:1111.3702.

[9] Liu, L., Moriyama, T., Ralph, D. C., Buhrman, R. A., "Spin-Torque Ferromagnetic Resonance Induced by the Spin Hall Effect," Phys. Rev. Lett. 106(3), 036601 (2011).

[10] Mosendz, O., Vlaminck, V., Pearson, J. E., Fradin, F. Y., Bauer, G. E. W., Bader, S. D., Hoffmann, A., "Detection and quantification of inverse spin Hall effect from spin pumping in permalloy/normal metal bilayers," Phys. Rev. B 82(21), 214403 (2010).

[11] Nakayama, H., Ando, K., Harii, K., Yoshino, T., Takahashi, R., Kajiwara, Y., Uchida, K., Fujikawa, Y., Saitoh, E., “Geometry dependence on inverse spin Hall effect induced by spin pumping in $\mathrm{Ni}_{81} \mathrm{Fe}_{19} / \mathrm{Pt}$ films," Phys. Rev. B 85(14), 144408 (2012).

[12] Obstbaum, M., Härtinger, M., Bauer, H. G., Meier, T., Swientek, F., Back, C. H., Woltersdorf, G., "Inverse spin Hall effect in $\mathrm{Ni}_{81} \mathrm{Fe}_{19} /$ normal-metal bilayers,” Phys. Rev. B 89(6), 060407 (2014).

[13] Vlaminck, V., Pearson, J. E., Bader, S. D., Hoffmann, A., "Dependence of spin-pumping spin Hall effect measurements on layer thicknesses and stacking order," Phys. Rev. B 88(6), 064414 (2013).

[14] Rojas-Sánchez, J.-C., Reyren, N., Laczkowski, P., Savero, W., Attané, J.-P., Deranlot, C., Jamet, M., George, J.-M., Vila, L., et al., "Spin Pumping and Inverse Spin Hall Effect in Platinum: The Essential Role of Spin-Memory Loss at Metallic Interfaces," Phys. Rev. Lett. 112(10), 106602 (2014).

[15] Althammer, M., Meyer, S., Nakayama, H., Schreier, M., Altmannshofer, S., Weiler, M., Huebl, H., Geprägs, S., Opel, M., et al., "Quantitative study of the spin Hall magnetoresistance in ferromagnetic insulator/normal metal hybrids," Phys. Rev. B 87(22), 224401 (2013). 
[16] Castel, V., Vlietstra, N., van Wees, B. J., Youssef, J. Ben., "Frequency and power dependence of spin-current emission by spin pumping in a thin-film YIG/Pt system," Phys. Rev. B 86(13), 134419 (2012).

[17] Nakayama, H., Althammer, M., Chen, Y.-T., Uchida, K., Kajiwara, Y., Kikuchi, D., Ohtani, T., Geprägs, S., Opel, M., et al., "Spin Hall Magnetoresistance Induced by a Nonequilibrium Proximity Effect," Phys. Rev. Lett. 110(20), 206601 (2013).

[18] Vlietstra, N., Shan, J., Castel, V., Ben Youssef, J., Bauer, G. E. W., van Wees, B. J., "Exchange magnetic field torques in YIG/Pt bilayers observed by the spin-Hall magnetoresistance," Appl. Phys. Lett. 103(3), 032401 (2013).

[19] Mosendz, O., Vlaminck, V., Pearson, J. E., Fradin, F. Y., Bauer, G. E. W., Bader, S. D., Hoffmann, A., "Detection and quantification of inverse spin Hall effect from spin pumping in permalloy/normal metal bilayers," Phys. Rev. B 82(21), 214403 (2010).

[20] Kimura, T., Hamrle, J., Otani, Y., "Estimation of spin-diffusion length from the magnitude of spin-current absorption: Multiterminal ferromagnetic/nonferromagnetic hybrid structures," Phys. Rev. B 72(1), 014461 (2005).

[21] Nguyen, H. Y. T., Pratt, W. P., Bass, J., “Spin-flipping in Pt and at Co/Pt interfaces,” J. Magn. Magn. Mater. 361, 30-33, Elsevier (2014).

[22] Liu, Y., Yuan, Z., Wesselink, R. J. H., Starikov, A. A., Kelly, P. J., "Interface enhancement of Gilbert damping from firstprinciples," arXiv:1406.6225.

[23] Fuchs, K., Mott, N. F., "The conductivity of thin metallic films according to the electron theory of metals," Math. Proc. Cambridge Philos. Soc. 34(01), 100-108 (1938).

[24] Castel, V., Vlietstra, N., Ben Youssef, J., van Wees, B. J., "Platinum thickness dependence of the inverse spin-Hall voltage from spin pumping in a hybrid yttrium iron garnet/platinum system," Appl. Phys. Lett. 101(13), 132414 (2012).

[25] Van der Pauw, L. J., "A Method of Measuring the Resistivity and the Hall Coefficient on Lamellae of Arbitrary Shape," Philips Tech. Rev. 26(8), 220-224.

[26] Hahn, C., de Loubens, G., Klein, O., Viret, M., Naletov, V. V., Ben Youssef, J., "Comparative measurements of inverse spin Hall effects and magnetoresistance in YIG/Pt and YIG/Ta," Phys. Rev. B 87(17), 174417 (2013).

[27] Ghosh, A., Auffret, S., Ebels, U., Bailey, W. E., "Penetration Depth of Transverse Spin Current in Ultrathin Ferromagnets," Phys. Rev. Lett. 109(12), 127202 (2012).

[28] Feng, Z., Hu, J., Sun, L., You, B., Wu, D., Du, J., Zhang, W., Hu, A., Yang, Y., et al., "Spin Hall angle quantification from spin pumping and microwave photoresistance," Phys. Rev. B 85(21), 214423 (2012).

[29] Mosendz, O., Pearson, J. E., Fradin, F. Y., Bauer, G. E. W., Bader, S. D., Hoffmann, A., "Quantifying Spin Hall Angles from Spin Pumping: Experiments and Theory,” Phys. Rev. Lett. 104(4), 046601 (2010).

[30] Azevedo, A., Vilela-Leão, L. H., Rodríguez-Suárez, R. L., Lacerda Santos, A. F., Rezende, S. M., "Spin pumping and anisotropic magnetoresistance voltages in magnetic bilayers: Theory and experiment," Phys. Rev. B 83(14), 144402 (2011).

[31] Tserkovnyak, Y., Brataas, A., Bauer, G. E. W., Halperin, B. I., "Nonlocal magnetization dynamics in ferromagnetic heterostructures," Rev. Mod. Phys. 77(October), 1375-1421 (2005).

[32] Jiao, H., Bauer, G. E. W., "Spin Backflow and ac Voltage Generation by Spin Pumping and the Inverse Spin Hall Effect," Phys. Rev. Lett. 110(21), 217602 (2013).

[33] Ando, K., Takahashi, S., Ieda, J., Kajiwara, Y., Nakayama, H., Yoshino, T., Harii, K., Fujikawa, Y., Matsuo, M., et al., "Inverse spin-Hall effect induced by spin pumping in metallic system," J. Appl. Phys. 109(10), 103913 (2011).

[34] Valet, T., Fert, A., "Theory of the perpendicular magnetoresistance in magnetic multilayers," Phys. Rev. B 48(10), 7099 (1993).

[35] Bass, J., Pratt, W. P., "Spin-diffusion lengths in metals and alloys, and spin-flipping at metal/metal interfaces: an experimentalist's critical review," J. Phys. Condens. Matter 19(18), 183201 (2007).

[36] Stiles, M., Zangwill, A., “Anatomy of spin-transfer torque,” Phys. Rev. B 66(1), 014407 (2002).

[37] Ghosh, A., Auffret, S., Ebels, U., Wilhelm, F., Rogalev, A., Bailey, W. E., "Effect of direct exchange on spin current scattering in Pd and Pt," Mesoscale and Nanoscale Physics, arXiv:1308.0450.

[38] Huang, S. Y., Fan, X., Qu, D., Chen, Y. P., Wang, W. G., Wu, J., Chen, T. Y., Xiao, J. Q., Chien, C. L., "Transport Magnetic Proximity Effects in Platinum," Phys. Rev. Lett. 109(10), 107204 (2012).

[39] Niimi, Y., Morota, M., Wei, D. H., Deranlot, C., Basletić, M., Hamzić, A., Fert, A., Otani, Y., "Extrinsic Spin Hall Effect Induced by Iridium Impurities in Copper," Phys. Rev. Lett. 106(12), 126601 (2011).

[40] Eid, K., Portner, D., Borchers, J., Loloee, R., Al-Haj Darwish, M., Tsoi, M., Slater, R., O’Donovan, K., Kurt, H., et al., “Absence of mean-free-path effects in the current-perpendicular-to-plane magnetoresistance of magnetic multilayers," Phys. Rev. B 65(5), 054424 (2002).

[41] Kurt, H., Loloee, R., Eid, K., Pratt, W. P., Bass, J., "Spin-memory loss at $4.2 \mathrm{~K}$ in sputtered $\mathrm{Pd}$ and $\mathrm{Pt}$ and at $\mathrm{Pd} / \mathrm{Cu}$ and $\mathrm{Pt} / \mathrm{Cu}$ interfaces," Appl. Phys. Lett. 81(25), 4787 (2002).

[42] Sharma, A., Romero, J. A., Theodoropoulou, N., Loloee, R., Pratt, W. P., Bass, J., "Specific resistance and scattering asymmetry of Py/Pd, Fe/V, Fe/Nb, and Co/Pt interfaces," J. Appl. Phys. 102(11), 113916 (2007). 